\title{
Research article \\ Electromyography changes in assessment of functional status of muscles in subjects with knee-osteoarthritis undergoing low level laser therapy
}

\author{
Arun Kumar M. ${ }^{1}$, Venkatesh D. ${ }^{2}$, Savita Ravindra ${ }^{3}$ \\ ${ }^{1}$ Assistant Professor, ${ }^{2}$ Professor, Department of Physiology, ${ }^{3}$ Professor and Head, Department of Physiotherapy, M S Ramaiah \\ Medical College, Bangalore, India
}

(Received: June $2020 \quad$ Revised: February $2021 \quad$ Accepted: March 2021)

Corresponding author: Arun Kumar M. Email: drarunkm@gmail.com

\begin{abstract}
Introduction and Aim: Knee Osteoarthritis (KOA) is a common degenerative joint disease which is one of the leading causes of disability in elderly people. Electromyography (EMG) is an electrophysiological method in evaluating skeletal muscle activity. Low-level laser (light) therapy (LLLT) is a modality of treatment used in several conditions required to suppress the pain, inflammation, stimulation of healing and restoration of function. Surface EMG parameters were studied before and after the low level laser therapy in subjects with knee osteoarthritis.

Materials and Methods: Subjects with knee OA participated in the study. Low level laser therapy (LLLT) was administered using a laser device with probe giving maximum power output of $10 \mathrm{mw}$ with a wavelength of $810 \mathrm{~nm}$. Surface electromyography (sEMG) of quadriceps muscles was recorded in all the study participants before and after the therapy. The parameters were statistically compared.
\end{abstract}

Results: There was a statistically significant difference between the maximum contraction and duration of contraction before and after the laser intervention in all the muscles.

Conclusion: It can be concluded that the muscle performance increased in the subjects with knee OA after the LLLT.

Keywords: Low Level Laser therapy (LLLT); knee osteoarthritis; electromyography; maximum amplitude of contraction.

\section{INTRODUCTION}

$\mathrm{K}$ nee Osteoarthritis (KOA) is a common degenerative joint disease which is one of the leading causes of disability in elderly people. Incidence of KOA is more as the age increases. The incidence is as high as $25 \%$ of the population in people over 55 years of age (1). The risk factors for KOA are several which include higher age, obesity, repetitive joint trauma, squatting and kneeling. Patients with KOA have severe pain and stiffness. Subjects suffering from KOA have profound effect on physical and psychological quality of life. Their mobility is restricted affecting normal domestic activities and adversely impacting overall normal lifestyle (2). KOA is known to occur because of wear and tear of the cartilage and the damage is extended to other parts of the joints like the meniscus and articular cartilage. There are several pathophysiological derangements which lead to the destruction of joint structure and thus worsening the disease. The conventional radiographs are widely considered as gold standard for the assessment of knee OA $(3,4)$.

Electromyography (EMG) is an electrophysiological method in recording and evaluating the skeletal muscle electrical activity. Needle EMG is the technique were the needle electrodes are inserted into the muscle of interest and activity are recorded. In surface electromyography (sEMG), the electrodes are placed on the skin surface over the muscle of interest and its activity is recorded. sEMG signals are affected by instrumentation, the amplifiers, neural control of the muscle and also the properties of the muscles itself (5). EMG amplitude and frequency are used to describe the recorded signal from the muscle. Amplitude of sEMG depends on the size, number and firing rate of motor units. It also describes the quantum of force generated in the muscle during activity and rest. Frequency of sEMG depends on the active firing rate, conduction velocity and number of active muscle fibres (6). sEMG is used in clinical assessment and prognosis of several conditions like neuromuscular disorders, Parkinson's disease, gait abnormalities and muscular rehabilitation. sEMG is recorded to quantify the muscle performance and the following parameters are used like maximum amplitude of contraction (MAC) of the muscle, number of maximum contraction and time taken for maximum contraction $(7,8)$.

Low-level laser (light) therapy (LLLT) is a modality of treatment used in several conditions required to suppress the pain, inflammation, stimulation of healing and restoration of function. In addition, it augments the tissue repair, promotes regeneration of tissues preventing further tissue damage (9). LLLT acts on the mitochondria to increase adenosine triphosphate (ATP) production, modulation of reactive oxygen species (ROS), and the induction of transcription factors (10). LLLT is known to be safe 
effective method in management of pain and inflammation in KOA (11).

In this study, surface EMG parameters were studied before and after the low level laser therapy in subjects with knee osteoarthritis to assess the impact of the therapy/intervention.

\section{MATERIALS AND METHODS}

The study was done in M S Ramaiah Medical College and Teaching Hospitals. Study participants were in the age group of 45-75 years who had clinically diagnosed knee osteoarthritis. Forty-five symptomatic subjects in Grade II and III of radiological criteria based on Kellgren-Lawrence (KL) grade II and III were included for the study. Subjects with KL grade I and IV, infective arthritis, history of vascular diseases in the lower limb, photosensitivity, and patient on steroid therapy were excluded from the study. Institutional scientific and ethics committee approval was obtained. Procedure was explained to the participants and written informed consent was obtained.

Surface electromyography (sEMG) of all the study participants was done using RMS electromyography equipment. Subjects were made to sit comfortably and recording was done using surface electrodes by placing the three leads around the knee joint. Each lead consists of three electrodes: recording electrode, the reference electrode and ground electrode. Recording electrodes were placed on the skin over the muscle's rectus femoris (Lead I), vastus lateralis (Lead II), and vastus medialis (Lead III). The reference electrodes were placed at the distance of $5 \mathrm{~cm}$ from recording electrode. The ground electrode was placed on the posterior surface of the knee joint. After the placement of the electrode, instructions were given to the patient to extend the leg at the knee joint and the recording was done. It was repeated at-least three times and the best attempt was considered for analysis of the signals. The onset of contraction, end of contraction, duration of contraction, maximum amplitude of contraction, and number of peaks during contraction of the muscle were calculated and tabulated. Analysis of the EMG signals were done.

Low level laser therapy (LLLT) was administered using a laser device with a probe giving maximum power output of $100 \mathrm{~mW}$ with a wavelength of 810 $\mathrm{nm}$. LLLT was given with the laser probe at 6 points around the surface of the affected knee joint. The dosage at each point was $1.5 \mathrm{~J}$ for a duration of 60 seconds The total dosage administered per session was $12 \mathrm{~J}$. The therapy was given on alternate days or 3 times a week for the duration of 3 months. Patients having involvement of both knee joints were given therapy for both the limbs accordingly.

The baseline parameters of the participants were mentioned as Mean \pm SD in the study. The sEMG parameters were compared before and after the completion of therapy using paired $t$ test. Pearson correlation was done between the baseline parameters and sEMG parameters. $\mathrm{p}<0.05$ was considered to be statistically significant.

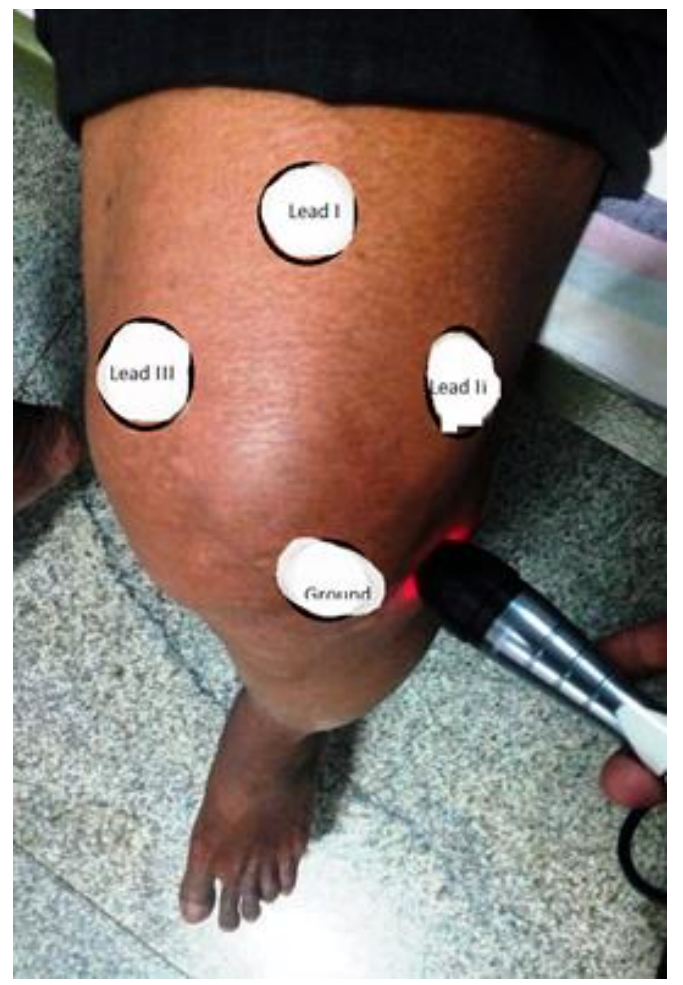

Fig. 1: Placement of electrodes for surface electromyography recording

\section{RESULTS}

The mean age of participants was $62.24 \pm 7.51$ years. The average weight was $71.53 \pm 10.42 \mathrm{~kg}$, height was $162.42 \pm 5.95 \mathrm{~cm}$. The mean BMI of the participants was $27.07 \pm 3.88 \mathrm{~kg} / \mathrm{m}^{2}$. The average duration of knee osteoarthritis in the study group was $6.51 \pm 3.35$ years (table 1).

Table 1: Baseline parameters of the study participants

\begin{tabular}{|c|c|}
\hline Parameters & Mean and SD \\
\hline Age in Years & $62.24 \pm 7.51$ \\
\hline Weight in Kg & $71.53 \pm 10.42$ \\
\hline Height in cm & $162.42 \pm 5.95$ \\
\hline BMI kgm $^{-2}$ & $27.07 \pm 3.88$ \\
\hline Duration of Knee OA in years & $6.51 \pm 3.35$ \\
\hline
\end{tabular}

The comparison of time of onset of contraction, end of contraction, duration of contraction before and after the LLLT was done using paired t tests. There was no significant statistical difference in mean time for onset of contraction. It was $1.811 \pm 0.695$ and $1.834 \pm 0.959$ seconds between the pre-intervention and postintervention group respectively. There was a statistically significant difference between the end of contraction and duration of contraction of the muscles of lead I before and after the laser intervention (table 2). Similarly, in Lead II and Lead III there was no significant difference in the onset of muscle contraction however there was significant difference for the end of contraction in the subjects after intervention (table 2, 3). 
Table 2: Comparison of duration of muscle contraction for Lead I (Rectus Femoris) before and after intervention in study participants

\begin{tabular}{|c|c|ccc|ccc|c|}
\hline Lead I & $\begin{array}{c}\text { Muscle contraction } \\
\text { (seconds) }\end{array}$ & \multicolumn{3}{|c|}{ Pre- intervention } & \multicolumn{2}{c|}{ Post -intervention } & p value \\
\hline \multirow{3}{*}{ Left } & Onset & 1.811 & \pm & 0.695 & 1.834 & \pm & 0.959 & 0.895 \\
\cline { 2 - 9 } & End & 5.723 & \pm & 1.782 & 7.118 & \pm & 1.517 & $0.000^{*}$ \\
\cline { 2 - 9 } & Duration & 3.912 & \pm & 1.711 & 5.285 & \pm & 1.448 & $0.000^{*}$ \\
\hline \multirow{2}{*}{ Right } & Onset & 2.119 & \pm & 0.904 & 1.955 & \pm & 0.768 & 0.353 \\
\cline { 2 - 9 } & End & 6.231 & \pm & 2.025 & 7.459 & \pm & 1.472 & $0.001^{*}$ \\
\cline { 2 - 9 } & Duration & 4.113 & \pm & 1.704 & 5.504 & \pm & 1.344 & $0.000^{*}$ \\
\hline
\end{tabular}

$* \mathrm{p}<0.05$ is considered statistically significant

Table 3: Comparison of duration of muscle contraction for Lead II (Vastus Lateralis) before and after intervention in study participants

\begin{tabular}{|c|c|ccc|ccc|c|}
\hline \multirow{2}{*}{ Lead II } & \multicolumn{2}{|c|}{$\begin{array}{c}\text { Muscle } \\
\text { Contraction (seconds) }\end{array}$} & \multicolumn{2}{|c|}{ Pre- intervention } & \multicolumn{2}{c|}{ Post -intervention } & p value \\
\hline \multirow{3}{*}{ Left } & Onset & 2.006 & \pm & 0.775 & 1.640 & \pm & 0.818 & 0.050 \\
\cline { 2 - 9 } & End & 6.542 & \pm & 1.763 & 7.342 & \pm & 1.609 & $0.016^{*}$ \\
\cline { 2 - 9 }$y$ & Duration & 4.535 & \pm & 1.495 & 5.701 & \pm & 1.417 & $0.000^{*}$ \\
\hline \multirow{3}{*}{ Right } & Onset & 1.828 & \pm & 0.786 & 1.564 & \pm & 0.485 & 0.052 \\
\cline { 2 - 9 } & End & 7.025 & \pm & 1.671 & 7.528 & \pm & 1.402 & 0.070 \\
\cline { 2 - 9 } & Duration & 5.198 & \pm & 1.515 & 5.964 & \pm & 1.514 & $0.008^{*}$ \\
\hline
\end{tabular}

$* \mathrm{p}<0.05$ is considered statistically significant

Table 4: Comparison of duration of muscle contraction for Lead III (Vastus Medialis) before and after intervention in study participants

\begin{tabular}{|c|c|ccc|ccc|c|}
\hline Lead III & $\begin{array}{c}\text { Muscle Contraction } \\
\text { (seconds) }\end{array}$ & \multicolumn{2}{|c|}{ Pre- intervention } & \multicolumn{2}{|c|}{ Post -intervention } & p value \\
\hline \multirow{3}{*}{ Left } & Onset & 1.757 & \pm & 0.911 & 1.527 & \pm & 0.906 & 0.193 \\
\cline { 2 - 9 } & End & 6.545 & \pm & 1.356 & 7.300 & \pm & 1.494 & $0.010^{*}$ \\
\cline { 2 - 9 } & Duration & 4.788 & \pm & 1.099 & 5.773 & \pm & 1.228 & $0.000^{*}$ \\
\hline \multirow{3}{*}{ Right } & Onset & 2.085 & \pm & 1.005 & 1.889 & \pm & 0.812 & 0.306 \\
\cline { 2 - 9 } & End & 6.610 & \pm & 1.969 & 7.887 & \pm & 1.325 & $0.001^{*}$ \\
\cline { 2 - 8 } & Duration & 4.526 & \pm & 1.508 & 5.998 & \pm & 1.151 & $0.000^{*}$ \\
\hline
\end{tabular}

$* \mathrm{p}<0.05$ is considered statistically significant

Duration of contraction of muscle in all the leads was significantly increased after the intervention in the study participants (Table 2, 3, 4; Fig. 1).

There was a statistically significant difference in the maximum amplitude of contraction in all the muscles before and after the intervention (fig. 2). There was a significant difference in the number of maximum amplitudes of contraction lead I and III but not in lead II before and after the LLLT (fig. 3).

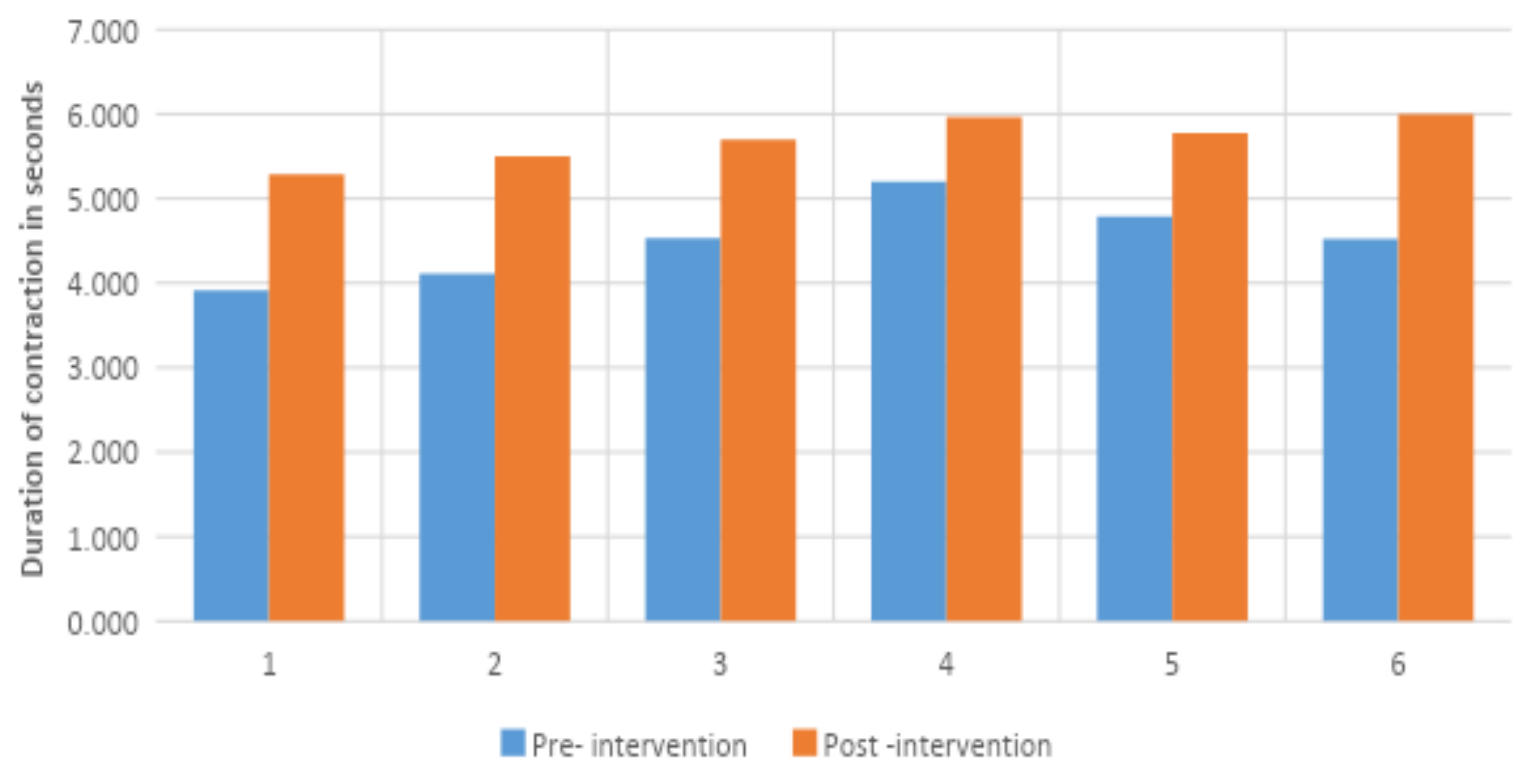

Fig. 2: Comparison of duration of muscle contraction before and after intervention (LLLT) in study participants 


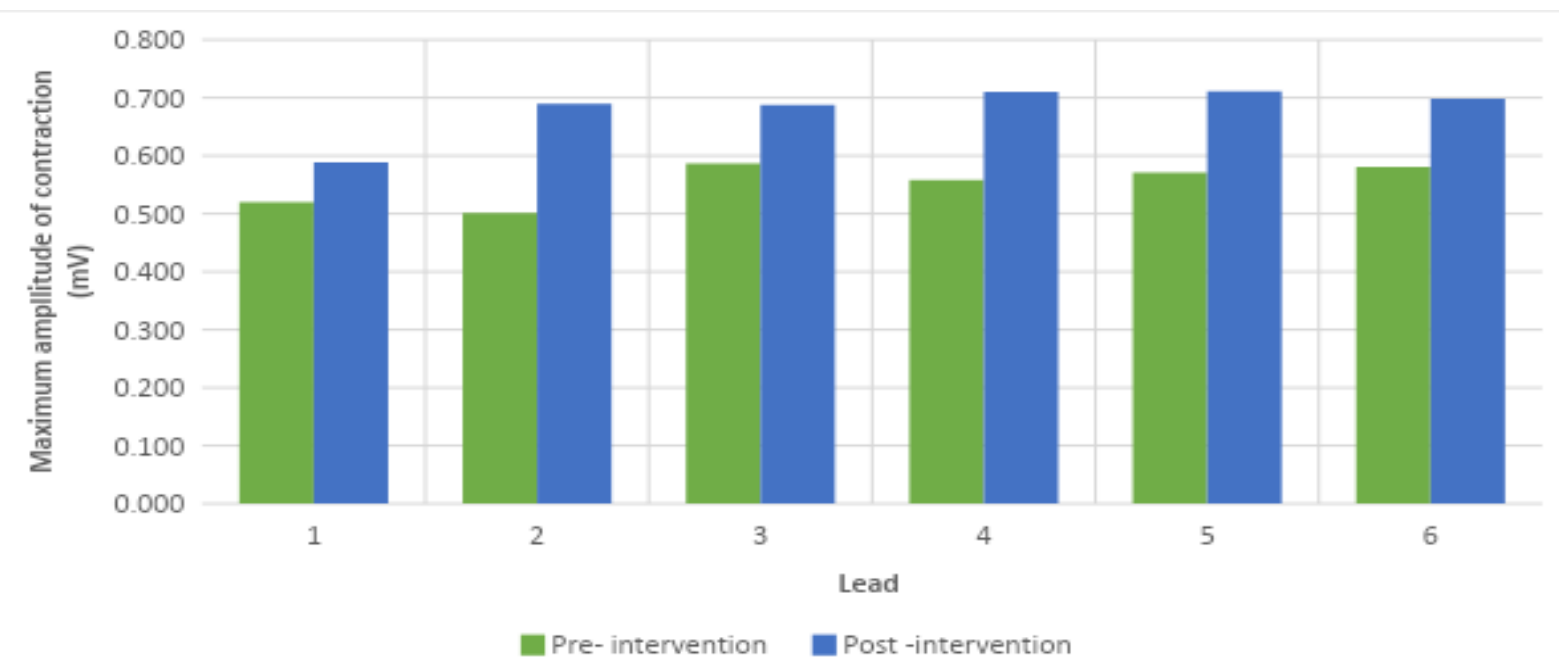

Fig. 3: Comparison of amplitude of muscle contraction in all leads before and after intervention (LLLT)

Table 5: Comparison of the number of maximum amplitudes of contraction (MAC) before and after intervention (LLLT).

\begin{tabular}{|c|c|ccc|ccc|c|}
\hline & Number of MAC $(\mathbf{m V})$ & \multicolumn{3}{|c|}{ Pre- intervention } & \multicolumn{3}{|c|}{ Post -intervention } & p value \\
\hline Lead I & Left & 10.156 & \pm & 6.613 & 15.733 & \pm & 6.760 & $0.000^{*}$ \\
\hline & Right & 8.222 & \pm & 5.125 & 13.511 & \pm & 6.348 & $0.000^{*}$ \\
\hline Lead II & Left & 12.133 & \pm & 7.095 & 15.333 & \pm & 8.796 & 0.065 \\
\hline & Right & 12.689 & \pm & 4.898 & 14.289 & \pm & 6.189 & 0.121 \\
\hline Lead III & Left & 11.644 & \pm & 4.349 & 16.200 & \pm & 7.424 & $0.000^{*}$ \\
\hline & Right & 12.267 & \pm & 5.614 & 15.378 & \pm & 5.245 & $0.015^{*}$ \\
\hline
\end{tabular}

$* \mathrm{p}<0.05$ is considered statistically significant

\section{DISCUSSION}

The performance of muscles around the knee joint was studied using the surface electromyography. The muscles studied were rectus femoris, vastus lateralis and vastus medialis in the study participants. Study participants were subjects with knee osteoarthritis who underwent low level laser therapy (LLLT) for 3 months. The EMG parameters studied were time parameters like onset, end and duration of contraction. The amplitude parameters were maximum voluntary contraction (MVC) and number of such MVC. These parameters were measured before and after LLLT intervention.

The lead I, Lead II and Lead III recorded the electrical activity from rectus femoris, vastus lateralis and vastus medialis respectively. Onset of contraction for all muscles were almost the same and there was no difference between pre-intervention and postintervention values. This is suggestive that the intervention has not affected the onset of muscle activity. End of contraction time was more for the post -intervention group in all the leads. And hence duration of contraction was more for the muscle after the intervention. This is suggesting that duration of muscle performance increased after the intervention.

Maximum amplitude of contraction is suggestive of strength of muscle contraction. There was an increase in the maximum amplitude of contraction after LLLT. This is suggestive that muscle strength had increased after the intervention. The number of MAC's is the number of such maximum contraction which was above the $50 \%$ of amplitude of maximum contraction. This is suggestive of number of active motor units in the muscle. There was an increase in such actively contracting motor units in the muscle which is indicative of increased muscle fibre activity for the same action performed before and after LLLT intervention. The results have demonstrated that there was an increase in the muscle performance after the intervention with low level laser therapy. According to Rice et al., there exists a muscle weakness and reduced co-activation of muscle fibres which leads to the loss of power of quadriceps muscle power in subjects with knee OA (12).

A study by Reid et al., the relationships between muscle strength of lower extremity and perceived disease severity in participants with knee osteoarthritis (OA) was studied. Muscle power was studied using the sEMG recording with MAC. it was reported that muscle power is an independent determinant of pain and quality of life in knee OA (13).

In a study by Booji et al., the muscle activation patterns and co-contraction of muscles of the knee joint was reported. This was done in patients with medial compartment knee OA response to walking with modified gait patterns. It was reported that the muscles showed increased flexor activation (24\%), cocontraction (17\%) and knee flexion moment (35\%). This could probably lead to increased load on knee muscles contributing to the frequent damage of the muscles (14). 
The patients with knee osteoarthritis have symptoms like pain and stiffness. Initially before the treatment was started patients had increased severity of pain and stiffness. This had reduced the routine activity and hence the performance of the muscle. This was also evident by increases in the subjective scores like Western Ontario and McMaster Universities Arthritis Index (WOMAC) and Lequesne severity index for knee OA. However, after the LLLT therapy, there was a reduction in the intensity of pain. This facilitated people to walk regularly and strengthen the muscles around the joint. The reduction of pain and increase in activity had contributed significantly to the improvement of the performance of the muscle evident from the recorded EMG parameters.

LLLT actions on muscle can be explained as follows. It is known that muscle works by contraction and relaxation which involves the utilization of the energy derived from ATP (15). It is demonstrated that the rate of firing of muscles is directly proportional to the ATP consumption. On working continuously, muscle develops fatigue because of several factors like depletion of ATP, accumulation of muscle metabolites like lactic acid and ions like potassium. It is known that when LLLT acts on the muscle it causes vasodilation, activating the cellular mechanism to increase ATP production. This enhances the muscle performance and delay the onset of fatigue. It has been demonstrated that LLLT increases muscle performance, delays fatigue, and hastens repair of the damaged cartilage (16). LLLT acts at different levels on several steps like ATP production, phosphocreatine re-synthesis, and also lactate oxygenation in the mitochondria. It is also shown that LLLT of higher wavelength is more effective in improving the muscle performance because of its deeper penetrability and optimum energy transfer $(17,18)$.

The muscles are not activated properly in the knee OA due to pain and restriction of movements. When the person starts moving, there is an instability and muscles are overloaded and hence there is damage to the muscle fibres. Damage is a disruption in the myofibrils accompanied by inflammation at the site of injury and loss of contractile function. The regeneration of muscle tissue after injury is a process that usually occurs in six phases taking about 21 days. There is acute inflammation of the muscle during 2 days following the injury and activation of satellite cells (19). There is regeneration of muscle fibres through myoblasts and new fibrils fill up the gap at the injured site in 14 days. The scar reduces and connective tissue reduces and there is fusion of end of myofibrils by 21 days (20). The process of inflammation and repair is supported by LLLT as it is known to promote the healing of the tissues. The myofibrils gain strength quicker and adds on to the existing fibres. Strength of muscle fibre increases and their potential to work also increases (21). The overall muscle activity in supporting the gait and movements is increased by LLLT.

The low level lasers therapy is known to reduce to the pain and inflammation in several tissues (22). Photobiomodulation at an appropriate fluence, wavelength, power output and frequency of irradiation can generate bio-stimulative effects on the preosteoblast cells, thereby causing a shift in the cell to an activated stage in the cell cycle (23). LLLT done using the wavelength of $810 \mathrm{~nm}$ has better penetration into the deeper tissues, even reaching the cartilage. The energy is absorbed by the deeper tissues and this energy is used for the repairing of the damaged cells, hence helping the regeneration of the damaged cartilage. In this process, there is reduction of pain producing substances and also pro-inflammatory markers (24). Low level laser therapy basically prevents the release of prostaglandins and other inflammatory mediators thus, reducing pain perception (25). Other mechanism of action could be LLLT acts on sodium- potassium pump, alters the nerve excitation and conduction thus decreasing the pain perception. LLLT may stimulate the production of endogenous endorphins which act on the mu receptors and prevent the action of substance $P$, thus reducing pain. Thus LLLT improves the tissue healing in the individuals in addition to reducing pain.

\section{CONCLUSION}

It can be concluded that LLLT increases the duration and amplitude of the muscle contraction as supported by the changes in the EMG. This is suggestive that the muscle has gained significant strength after the LLLT. And also LLLT was shown to be beneficial in improving the daily activity of the individual. Electromyography seems to be a reliable tool in assessing the prognosis of knee OA undergoing low level laser therapy.

\section{ACKNOWLEDGEMENTS}

I sincerely acknowledge Dr Maruti, Professor of Physiology and also Department of Physiotherapy, M S Ramaiah Medical College for their support.

\section{CONFLICT OF INTEREST}

No conflicts of interest declared

\section{REFERENCES}

1. Hayami, T. Osteoarthritis of the knee joint as a cause of musculoskeletal ambulation disability symptom complex (MADS). Clin Calcium. 2008; 18: 1574-1580.

2. Heidari, B. Knee osteoarthritis prevalence, risk factors, pathogenesis and features: Part I. Caspian J Intern Med. 2011; 2(2): 205-212.

3. Wick, M. C., Kastlunger, M., Weiss, R. J. Clinical imaging assessments of knee osteoarthritis in the elderly: a mini-review. Gerontology. 2014; 60(5): 386-394.

4. Cushnaghan, J., Cooper, C., Dieppe, P., Kirwan, J., McAlindon, T., McCrae, F. Clinical assessment of osteoarthritis of the knee. Ann Rheum Dis. 1990 Oct; 49(10): 768-770. 
5. Mills, K. R. The basics of electromyography. Journal of Neurology, Neurosurgery \& Psychiatry. 2005 Jun 1; 76(suppl 2): 32-35.

6. Chowdhury, R. H., Reaz, M. B. I., Ali, M. A. B. M., Bakar, A. A. A., Chellappan, K., Chang, T. G. Surface electromyography signal processing and classification techniques. Sensors (Basel). 2013 Sep 17; 13(9): 1243112466.

7. Kendell, C., Lemaire, E. D., Losier, Y., Wilson, A., Chan, A., Hudgins, B. A novel approach to surface electromyography: an exploratory study of electrode-pair selection based on signal characteristics. J Neuroeng Rehabil. 2012 Apr 26; 9: 24.

8. Pullman, S. L., Goodin, D. S., Marquinez, A. I., Tabbal, S., Rubin, M. Clinical utility of surface EMG. Neurology. $2000 \mathrm{Jul}$ 25; 55(2): 171.

9. Avci, P., Gupta, A., Sadasivam, M., Vecchio, D., Pam, Z., Pam, N., et al., Low-level laser (light) therapy (LLLT) in skin: stimulating, healing, restoring. Semin Cutan Med Surg. 2013 Mar; 32(1): 41-52.

10. Chung, H., Dai, T., Sharma, S. K., Huang, Y. Y., Carroll, J. D., Hamblin, M. R. The nuts and bolts of low-level laser (light) therapy. Annals of Biomedical Engineering. 2012 Feb; 40(2): 516.

11. Hegedűs, B., Viharos, L., Gervain, M., Gálfi, M. The Effect of low-level laser in knee osteoarthritis: a doubleblind, randomized, placebo-controlled trial. Photomed Laser Surg. 2009 Aug;27(4):577-584.

12. Rice, D. A., McNair, P. J., Lewis, G. N. Mechanisms of quadriceps muscle weakness in knee joint osteoarthritis: the effects of prolonged vibration on torque and muscle activation in osteoarthritic and healthy control subjects. Arthritis Res Ther. 2011; 13(5): R151.

13. Reid, K. F., Price, L. L., Harvey, W. F., Driban, J. B., Hau, C., Fielding, R. A., et al., Muscle power is an independent determinant of pain and quality of life in knee osteoarthritis. Arthritis Rheumatol. 2015 Dec; 67(12): 3166-3173.

14. Booij, M. J., Richards, R., Harlaar, J., van den Noort, J. C. Effect of walking with a modified gait on activation patterns of the knee spanning muscles in people with medial knee osteoarthritis. Knee. 2019.

15. Christie, A. D., Foulis, S. A., Kent, J. A. ATP cost of muscle contraction is associated with motor unit discharge rate in humans. Neurosci Lett. 2016; 629: 186-188.

16. Karu, T. I. Mitochondrial mechanisms of photobiomodulation in context of new data about multiple roles of ATP. Photomed. Laser Surg. 2010; 28: 159-160.

17. Tripathi, T., Ganesh, G., Rai, P. Pain pathways and effect of low level laser therapy on pain during fixed orthodontic treatment-an overview. European Journal of Dentistry and Medicine. 2016; 8: 1-5.

18. Kingsley, J. D., Demchak, T., Mathis, R. Low-level laser therapy as a treatment for chronic pain. Front Physiol [Internet]. 2014 Aug 19; 5.

19. Kuang, S., Rudnicki, M. A. The emerging biology of satellite cells and their therapeutic potential. Trends Mol Med. 2008;14(2): 82-91.

20. Chargé, S. B., Rudnicki, M. A. Cellular and molecular regulation of muscle regeneration. Physiol Rev. 2004; 84(1): 209-238.

21. Hawke, T. J., Garry, D. J. Myogenic satellite cells: physiology to molecular biology. J Appl Physiol. 2001; 91(2): 534-51.

22. Hanna, R., Agas, D., Benedicenti, S., Ferrando, S., Laus, F., Cuteri, V., et al., A comparative study between the effectiveness of $980 \mathrm{~nm}$ photobiomodulation delivered by hand-piece with gaussian vs. flat-top profiles on osteoblasts maturation. Front Endocrinol (Lausanne) [Internet]. 2019 Feb 20 [cited 2019 May 22]; 10.

23. Cotler, H. B., Chow, R. T., Hamblin, M. R., Carroll, J. The Use of low level laser therapy (1llt) for musculoskeletal pain. MOJ Orthop Rheumatol. 2015; 2(5): 00068.
24. Dima, R., Tieppo Francio, V., Towery, C., Davani, S. Review of literature on low-level laser therapy benefits for nonpharmacological pain control in chronic pain and osteoarthritis. Altern Ther Health Med. 2018 Sep; 24(5): 8-10.

25. Wickenheisser, V. A., Zywot, E. M., Rabjohns, E. M., Lee, H. H., Lawrence, D. S., Tarrant, T. K. Laser light therapy in inflammatory, musculoskeletal, and autoimmune disease. Curr Allergy Asthma Rep. 2019 02; 19(8): 37. 